\title{
Comparative analysis of complete chloroplast genomes sequences of Arctium lappa and A. tomentosum
}

\author{
Y.-P. XING ${ }^{1}$, L. XU'*, S.-Y. CHEN ${ }^{1}$, Y.-M. LIANG ${ }^{1}$, J.-H. WANG ${ }^{1}$, C.-S. LIU², T. LIU², \\ and T.-G. KANG ${ }^{1 *}$ \\ College of Traditional Chinese Medicine, Liaoning University of Traditional Chinese Medicine, \\ Dalian 116600, P.R. China ${ }^{l}$ \\ College of Traditional Chinese Medicine, Beijing University of Chinese Medicine, Beijing 100102, P.R. China \\ School of Pharmacy, China Medical University, Shenyang 110122, P.R. China ${ }^{3}$
}

\begin{abstract}
Arctium lappa and A. tomentosum are known medicinal plants in China. The complete chloroplast genomes from A. lappa and $A$. tomentosum were sequenced using Illumina sequencing technology. The total genome sizes of the complete chloroplast genomes of A. lappa and A. tomentosum were $152767 \mathrm{bp}$ and $152688 \mathrm{bp}$, respectively, and contained a pair of inverted repeats of the same length $(15,181 \mathrm{bp})$. The small single-copies were $18584 \mathrm{bp}$ and $18582 \mathrm{bp}$, and the large single-copies were $83821 \mathrm{bp}$ and $83744 \mathrm{bp}$, respectively. We identified and annotated 134 and 126 genes from A. lappa and $A$. tomentosum including, respectively, 90 and 89 protein-coding genes, 36 and 29 tRNAs, and eight rRNAs. A. lappa was found to have 10 tRNAs different from those in A. tomentosum, and A.tomentosum had three tRNAs different from those in A. lappa. There were only two types of simple sequence repeats of two species, mononucleotide and dinucleotide, and the sequences were $\mathrm{A}$ and $\mathrm{T}$ rich. In addition, the two ways of phylogenetic analysis show that the position of $A$. lappa and A. tomentosum is consistent within Asteraceae.
\end{abstract}

Additional key words: medicinal plants, phylogenetic analysis, simple sequence repeats.

\section{Introduction}

There are approximately 11 species of Arctium (Asteraceae) in the world, and they are widely distributed in temperate regions of Eurasia. Arctium lappa L. and A. tomentosum Mill. are distributed in China and used as medicinal plants. Recent studies of Arctium from China have focused on medicinal quality, internal transcribed spacer sequence identification, chemical composition, and pharmacological effects (Kang et al. 2009, Xu et al. 2011), but limited research has been focused on chloroplast (cp) genome identification, phylogenetic position, and species diversity.

In general, the cp genomes of vascular plants share a similar structure and are highly conserved (Jeffrey et al. 1982, Jansen et al. 2005, Choi et al. 2015). The highly conservative structure of the cp genome makes it suitable for comparative analysis of distant and closely related species (Raubeson and Jansen 2005). The cp genome size varies between 120 and $220 \mathrm{~kb}$, with one inverted repeat (IR) region (20 - $28 \mathrm{~kb})$, a large single copy (LSC) region (80 - $90 \mathrm{~kb}$ ), and a small single copy (SSC) region (16 - $27 \mathrm{~kb}$ ) (Wicke et al. 2017). The cp genome usually contains about 110 - 130 genes, including about 79 protein coding genes, about 30 transfer RNA (tRNA) encoding genes, and about 4 ribosomal RNA (rRNA) encoding genes. They are mainly involved in photosynthesis or gene expression (Jansen et al. 2005, Yang et al. 2013). With the emergence and development of high-throughput sequencing technology, more and more $\mathrm{cp}$ genomes of Asteraceae species have been dissected recently. Previous studies have demonstrated that the Asteraceae cp genomes had a relatively conservative number of genes and the size of intergenic regions contributed mainly to the variation of cp genome size (Wang et al. 2015). In the Asteraceae, the cp genomes share a $22.8 \mathrm{~kb}$ large inversion and a $3.3 \mathrm{~kb}$

Submitted 1 March 2018, last revision 7 October 2018, accepted 11 December 2018.

Abbreviations: cp - chloroplast; IR - inverted repeat; LSC - large single copy; SNP - single nucleotide polymorphism; SSC - small single copy; SSR - simple sequence repeat; ML - maximum-likelihood.

Acknowledgements: This work was supported by the National Natural Science Foundation of China (General Program, Nos 81773852 and 81874338) and the Liaoning Province Education Department (Liaoning Higher School Outstanding Young Scholar Growth Plan, No. LJQ2014101).

*Corresponding authors e-mails: 861364054@qq.com, kangtingguo@163.com 
smaller inversion nested within it (Kim et al. 2005). Many genes from cp genomes, such as $p s b \mathrm{~A}-\operatorname{trn} \mathrm{H}, \operatorname{trn} \mathrm{C}-y c f 6$, $y c f 6-p s b \mathrm{M}$, trn $\mathrm{Y}-r p o \mathrm{~B}, r p s 4-t r n \mathrm{~T}, \operatorname{trn} \mathrm{L}-\mathrm{F}, r p l 16$, matK, and $r b c \mathrm{~L}$ have been used for analysis of the phylogeny of Chrysanthemum, A. lappa and other plants (Liu et al. 2012, Tseng et al. 2012, Kim et al. 2016). Simple sequence repeat (SSR) is a class of tandemly repeated sequences that consists of $1-6$ nucleotide repeat units. The SSRs are important in plant typing and widely developed as molecular genetic markers for species identification $(\mathrm{Wu}$ et al. 2018). The cp SSRs are also used to assess the molecular phylogeny within the family Cucurbitaceae and Compositae (Chung et al. 2003, Wills et al. 2005).

The aim of this study was to sequence whole cp genomes of A. lappa and A. tomentosum and characterize their structure and the differences between species. We then aimed to find the evolutionary relationships in the Arctium genus to identify appropriate regions or genes for use as markers.

\section{Materials and methods}

Plant materials: Fresh Arctium lappa leaves were collected from Dalian, China (E $121^{\circ} 52^{\prime}, \mathrm{N} 39^{\circ} 03^{\prime}$ ) and fresh $A$. tomentosum leaves were collected from Urumqi, China (E $\left.84^{\circ} 33^{\prime}, \mathrm{N} 44^{\circ} 07^{\prime}\right)$. Both were identified by Dr. T. Kang from the Liaoning University of Traditional Chinese Medicine in Shenyang, China. Voucher specimens were deposited in the Liaoning University of Traditional Chinese Medicine Herbarium (A. lappa 20170417005LY, A. tomentosum 20170616001LY).

Chloroplast DNA extraction and sequencing: Approximately $5 \mathrm{~g}$ of fresh leaves was harvested for chloroplast DNA isolation using a method of Mcpherson et al. (2013). Then, $1 \mu \mathrm{g}$ of purified DNA was fragmented and used to construct short-insert libraries (insert size $430 \mathrm{bp}$ ) according to the manufacturer's instructions (Illumina, USA), then sequenced on an Illumina Hiseq 4000 (Borgstrom et al. 2011).

Genomic assembly: Prior to assembly, raw reads were filtered in order to remove the reads with adaptors, the reads showing a quality score below $20(Q<20)$, the reads containing a percentage of uncalled bases (" $N$ " characters) equal or greater than $10 \%$, and the duplicated sequences. The $\mathrm{cp}$ genome was reconstructed using a combination of de novo and reference-guided assemblies, and the following three steps were used (Cronn et al. 2008, Koren et al. 2012): 1 ) the filtered reads were assembled into contigs using SOAP denovo 2.04 (Luo et al. 2012); 2) contigs were aligned to the reference genome of two species using $B L A S T$, and aligned contigs $(\geq 80 \%$ similarity and query coverage) were ordered according to the reference genome; and 3) clean reads were mapped to the assembled draft $\mathrm{cp}$ genome to correct the wrong bases, and the majority of gaps were filled through local assembly.

Genomic anotation and analysis: The cp genes were annotated using an online DOGMA tool (Wyman et al. 2012) using default parameters to predict genes encoding proteins, tRNA, and rRNA. Whole chloroplast genomes BLAST search (Altschul et al. 1990) (E-value $\leq 1 \mathrm{e}^{-5}$, a minimal alignment length $>40 \%$ ) was performed against five databases. They were $K E G G$ (Kyoto Encyclopedia of Genes and Genomes; Kanehisa et al. 1990, 1997, 2006), COG (Clusters of Orthologous Groups; Tatusov et al. 1997, 2003), NR (Non-Redundant Protein Database), Swiss-Prot (Magrane 2011), and GO (Gene Ontology; Ashburner et al. 2000). The SSR software Micro Satellite (MISA; http://pgrc.ipk-gatersleben.de/misa/) was used to identify the SSR sequences, and tandem repeats of 1 - 6 nucleotides were considered as microsatellites. The minimum numbers of repeats were set to $11,6,5,5,5$, and 5 for mono-, di-, tri-, tetra-, penta-, and hexa-nucleotides, respectively. The maximal number of bases interrupting 2 SSRs in a compound microsatellite was 100 . We focused on perfect repeat sequences. The mVISTA was used to analyze similarities between four Asteraceae species (Frazer et al. 2004). Web-based REPuter (http://bibiserv. techfak.uni-bielefeld.de/reputer/) was used to analyze the long repeat sequences, which included forward, reverse, and tandem repeats with a minimum sequence length of $30 \mathrm{bp}$ and edit distances of $3 \mathrm{bp}$.

Chloroplast genome mapping: The chloroplast genomes of $A$. lappa and $A$. tomentosum were exported in the GenBank format using the Sepquin software, and the cp genome was mapped using the annotation results (Wang et al. 2015) (http://ogdraw.mpimp-golm.mpg. de/index.shtml). Finally, the complete cp genomes were submitted to the NCBI GenBank database (accession number: MH375874).

Phylogenetic analysis: To identify the phylogenetic position of Arctium species within Asteraceae and their relationship to other families, phylogenetic trees were constructed by the cp genome sequences from 36 species, 34 of them were downloaded from GenBank. Among them, three species, Lathyrus clymenum, Arabidopsis thaliana, and Nicotiana tabacum, were set as outgroups. Thirty one Asteraceae species and two species from Araliaceae and Campanulaceae were also included in the phylogeny. The analysis was run using whole chloroplast genome single nucleotide polymorphisms (SNPs) and 25 genes were shared by all 36 species (Table 1 Suppl.). Maximum-likelihood (ML) methods were performed for the phylogenetic analyses using PhyML 3.0, and a model $G T R+I+G$ was selected for ML analyses with 100 bootstrap replicates to calculate bootstrap values.

\section{Results and discussion}

The total length of the chloroplast (cp) genomes of Arctium lappa and A. tomentosum were $152767 \mathrm{bp}$ and 152688 bp (Fig. 1A,B), respectively. This is similar to the cp genomes of other genera in Asteraceae, which were downloaded from GenBank indicating that the Asteraceae 


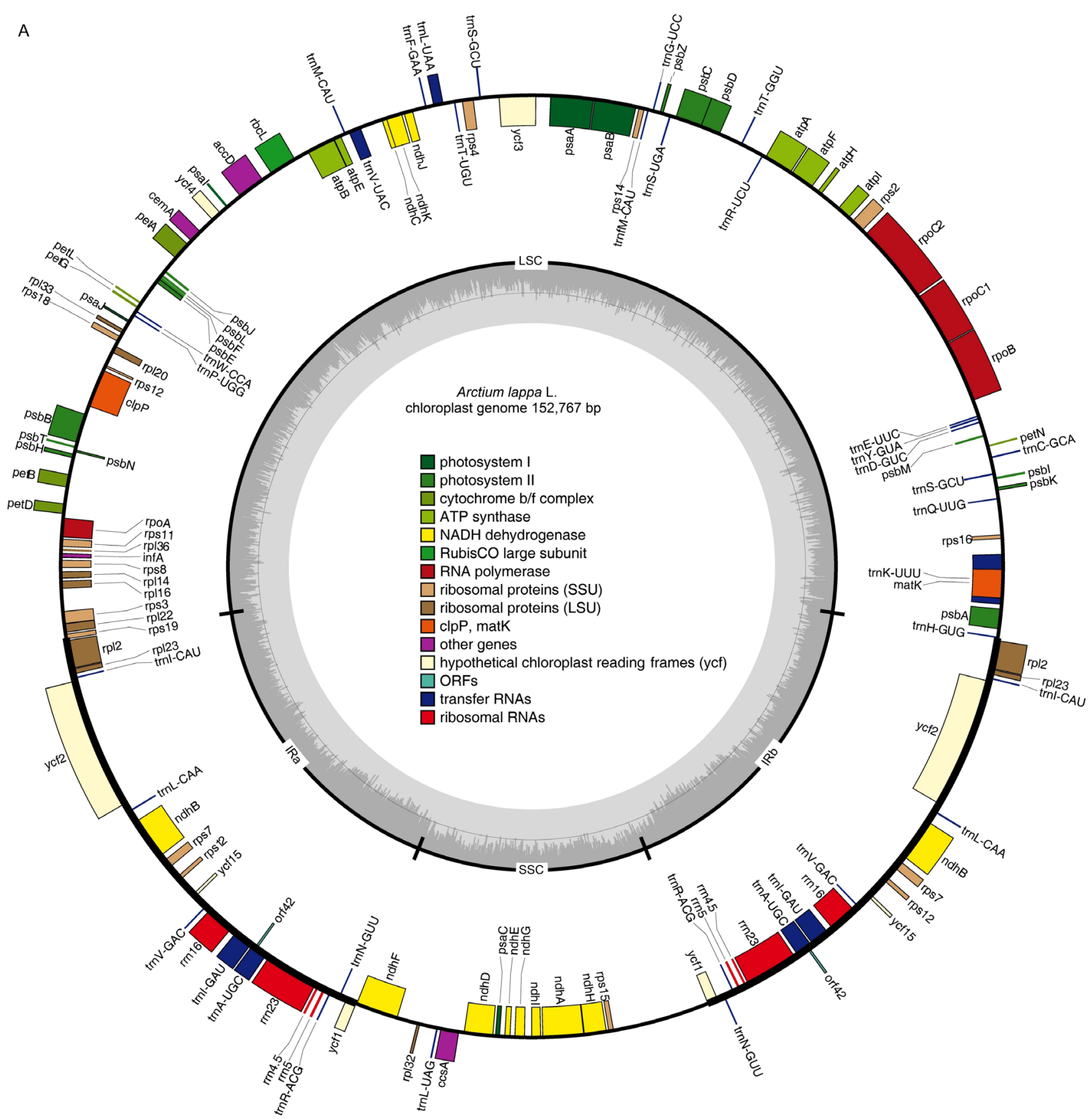

Fig. 1A. A chloroplast genome map illustrates Arctium lappa. The outer ring shows the gene, non-coding RNA and the positions of other genomic components corresponding to the gene name; the inner ring shows the genomic GC content.

cp genomes are very conservative ( $\mathrm{Li}$ et al. 2013). The LSC lengths of $A$. lappa and $A$. tomentosum were 83821 bp and $83744 \mathrm{bp}$, the SSC lengths were $18584 \mathrm{bp}$ and $18582 \mathrm{bp}$, the IR (IRa, IRb) region of them shared the same length of $25181 \mathrm{bp}$, and the GC content were 38.02 and $37.69 \%$, respectively (Table 1 ). There were 134 and 126 genes annotated for A. lappa and A. tomentosum, respectively.

Arctium lappa contained 36 tRNA genes, and 9 rRNA and 90 protein coding genes (Table 2 Suppl.). Fourteen tRNA genes and all rRNAs were located in the IR region
(Fig. 1A). Arctium tomentosum contained 89 protein coding genes, 29 tRNA genes, and 8 rRNA genes in the IR region, and 10 tRNAs in the IR region (Fig. 1B). Based on comparisons of published chloroplast genome sequences of Panax (NC_006290.1) (Kim et al. 2004), two inversions were present in the chloroplast genomes of A. lappa and A. tomentosum. One boarder of the larger inversion was located between the trnS-GCU and trnCGCA genes, and the other boarder was located between the $\operatorname{trn} \mathrm{T}-\mathrm{GCT}$ and $\operatorname{trn} \mathrm{R}-\mathrm{TCT}$ genes. The smaller inversion had one boarder located between the trnC-GCA and $r p o \mathrm{~B}$ 


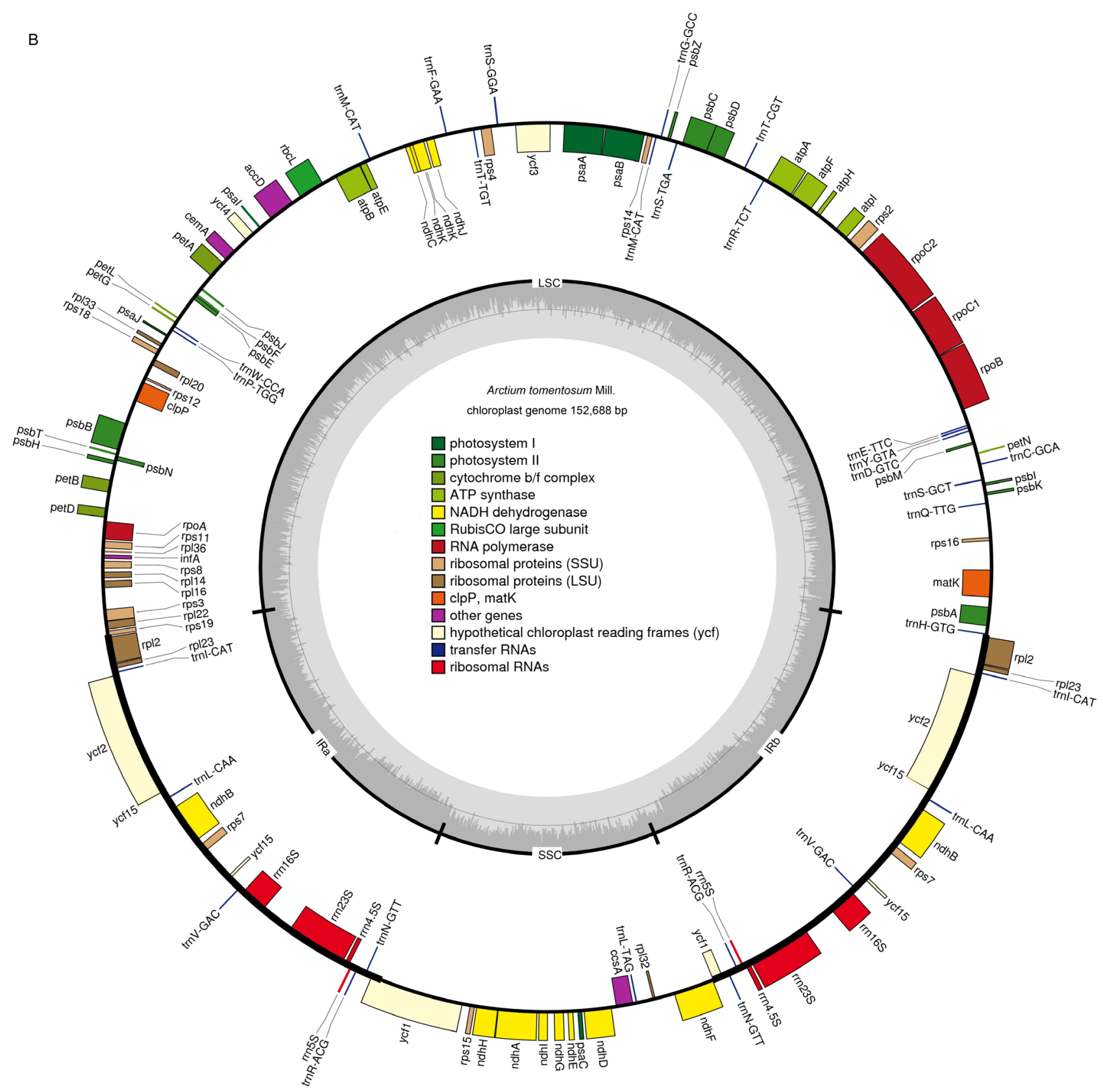

Fig. 1B. A chloroplast genome map illustrates Arctium tomentosum. The outer ring shows the gene, non-coding RNA and the positions of other genomic components corresponding to the gene name; the inner ring shows the genomic GC content.

genes and the other boarder between the $p s b \mathrm{M}$ and $t r n \mathrm{D}-$ GUC (Fig. 1 Suppl.).

The annotated genes from A. lappa and A. tomentosum (Table 2 Suppl.) were generally similar, but some differences were observed. Specifically, the matK gene in A. lappa was located in the intron of the trnK-UUU gene, whereas $A$. tomentosum had no trnK-UUU gene. There was specific trans-splicing in rps 12 of $A$. tomentosum, the 5 ' end of the exon was located in the LSC region and the $3^{\prime}$ end in the IR region, which is consistent with Magnolia grandiflora (Li et al. 2013) and ginseng (Kim et al. 2004).
The rps 12 was located in the IR region of A. lappa., which had more transfer RNA genes annotated: $\operatorname{trn} \mathrm{G}-\mathrm{UCC}$, $\operatorname{trn} \mathrm{I}-$

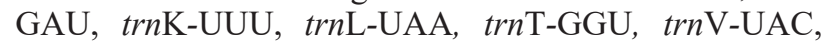
$\operatorname{trn} \mathrm{A}-\mathrm{UGC}$, and trnI-GAU with the first six located in the LSC region and the last two in the IR area. The genes trnSGGA, trnT-CGT, and trnG-GCC from the A. tomentosum were not annotaed in the $A$. lappa cp genome. In the subunits of photosystem II, the gene $p s b \mathrm{~L}$ was absent in the A. tomentosum. In the conserved hypothetical chloroplast reading genes, the $y c f 15$ gene was located in the IR region with two copies in each IR region of $A$. tomentosum, but 
Table 1. Comparison of general features of the chloroplast genomes in four Asteraceae species. LSC - large single-copies; SSC - small single copies; IR - inverted repeat.

\begin{tabular}{lcccc}
\hline Species & Arctium lappa & A.tomentosum & $\begin{array}{l}\text { Chrysanthemum } \times \\
\text { morifolium }\end{array}$ & C. indicum \\
\hline Gene length [bp] & 152767 & 152688 & 151003 & 151129 \\
GC content [\%] & 38.02 & 37.69 & 37.48 & 37.42 \\
LSC length [bp] & 83821 & 83744 & 82782 & 82810 \\
SSC length [bp] & 18584 & 18582 & 18354 & 18377 \\
IR length [bp] & 25181 & 25181 & 24953 & 24971 \\
Gene number & 134 & 126 & 129 & 129 \\
Gene number in IR regions & 42 & 36 & 17 & 17 \\
Protein-coding gene number & 90 & 89 & 85 & 85 \\
rRNA gene number & 8 & 8 & 36 & 36 \\
tRNA gene number & 36 & 29 & & 86 \\
\hline
\end{tabular}

it had one copy in each IR region of A. lappa. A. lappa had the putative genes orf42-D2 and orf 42 , which were not annotated for $A$. tomentosum. The genes $m a t \mathrm{~K}, \operatorname{trn} \mathrm{K}-$ UUU, trnS-GGA, trnT-CGT, trnG-GCC, and $p s b \mathrm{~L}$ could be used to develop molecular makers for the species.

Introns play an important role in the regulation of gene expressions. Some recent studies have found that many introns can improve the expression and timing of exogenous genes at specific locations, which may lead to the appearance of expected agronomic traits in genetically modified plants; therefore, introns can be useful tools for improving desired agronomic traits (Jiao et al. 2012). A. lappa chloroplast DNA had 10 coding genes with introns. Among them, the $c l p \mathrm{P}$ gene contained three introns and $y c f 3$ contained two introns. A. tomentosum chloroplast DNA had 11 genes containing introns. Among them, $y c f 1$ contained three introns, and $y c f 3$ contained two introns (Table 3 Suppl.). These introns may help to improve plant resilience and developing new cultivars.

Simple sequence repeats are effective molecular markers with a vast number of applications (Ravi et al. 2008, Kang et al. 2017). The SSRs are rich in quantity, co-dominant, and highly repetitive and also have a simple genomic structure, which is relatively conserved making them widely used for species identification and genetic analysis of individuals and groups (Khakhlova and Bock 2006, Curci 2015).

In the cp genomes of $A$. lappa and A. tomentosum, we found 16 and 24 SSRs, respectively (Table 2). The total lengths of SSRs in plastomes of $A$. lappa and A. tomentosum were 118 and 274 bp, respectively. There were only two types of SSRs (mononucleotides and dinucleotides). The dinucleotide SSRs of $A$. lappa were AT/AT and TA/TA repeats, and there were eight mononucleotide SSRs located in the LSC region. There was one mononucleotide SSR located in the SSC region and two located in the IR region. Arctium tomentosum had 19 SSRs located in the LSC region, 2 of which were dinucleotide SSRs, and 4 mononucleotide SSRs located in the IR region, and one mononucleotide SSR sequence located in the SSC region (Table 4 Suppl.) Both SSRs were A/T rich consistently with the $A$. lappa and $A$. tomentosum chloroplast genomes. We compared the abundance of the SSRs with two related species of $C$. (Fig. 2). When analyzed the SSR sequences of the four species of Asteraceae, all had more mononucleotide sequences and fewer trinucleotide sequences. Only $C$. indicum had a small amount of trinucleotide SSRs, and the SSRs of several plant species of Asteraceae were similar, which may provide information for finding SSR markers.

Large repeat sequences showed repeats with length $\geq 30$ bp each. Forty-six and 36 pairs of large repeat sequences with a sequence identity $>90 \%$ were found in the A. lappa (Table 5 Suppl.) and A. tomentosum cp genomes (Table 6 Suppl.). The repeats from $A$. lappa ranged from 30 to $116 \mathrm{bp}$ in length and from $A$. tomentosum ranged from 30 to $46 \mathrm{bp}$ in length. A total of 16 and 19 large repeat sequences were located in protein-coding genes in A. lappa and $A$. tomentosum, respectively. Thirty and 17 large repeat sequences were located in the intergenic regions

Table 2. Type and abundance of different single sequence repeats (SSR) in Arctium lappa and A. tomentosum.

\begin{tabular}{lllllcc}
\hline & A. lappa & & \multicolumn{1}{c}{ A. tomentosum } \\
& SSR repeats & SSR abundances & Percent abundance [\%] & SSR repeats & SSR abundances Percent abundance [\%] \\
\hline Mononucleotide & A/T & 12 & 75 & A/T & 21 & 87.5 \\
\multirow{5}{*}{ Dinucleotide } & G/C & 2 & 12.5 & G/C & 1 & 4.17 \\
& AT/AT & 1 & 6.25 & AT/AT & 2 & 8.33 \\
& TA/TA & 1 & 6.25 & - & - & - \\
\hline
\end{tabular}




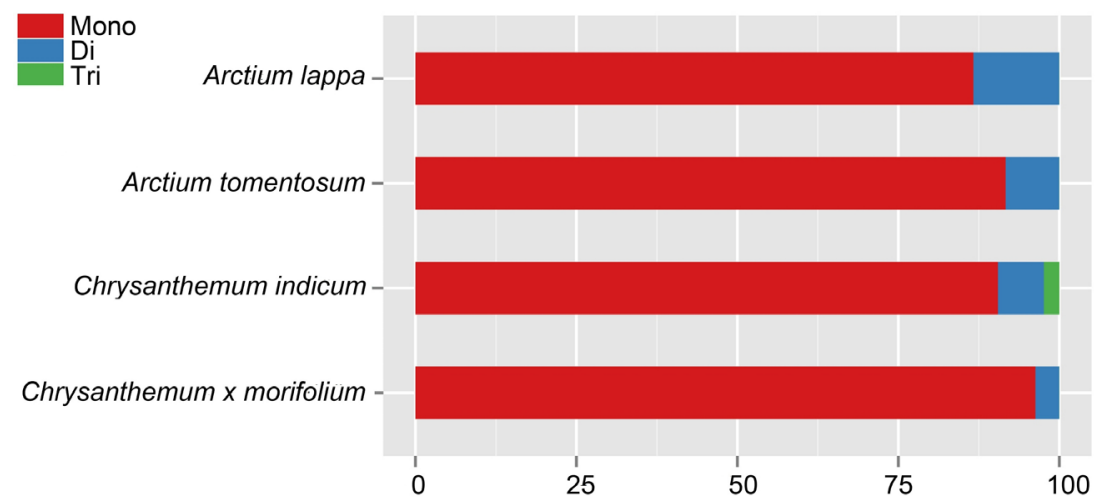

Fig. 2. Single sequence repeats in the chloroplast genomes of two species of Arctium and two species of Chrysanthemum. Mono represents mononucleotide repeats, Di represents dinucleotide repeats, and Tri represents trinucleotide repeats.Arctium spp. and Chrysanthemum spp.

in A. lappa and A. tomentosum, respectively. Numerous repeat sequences have been identified in the intergenic spacer regions in $\mathrm{cp}$ genomes of several angiosperm lineages (Yang et al. 2013).

Changes in genomic size are mainly due to differences in the length of LSC and IR regions (Chumley et al. 2006, $\mathrm{Wu}$ and Ge 2012). The length of the IR region in some species of Magnoliaceae have a positive relationship with the length of the complete $\mathrm{cp}$ genome sequence ( $\mathrm{Li}$ et al. 2013). By comparing the boundary characteristics of A. lappa, A. tomentosum, C. $\times$ morifolium, and C. indicum, we found that the length of the IR region was conservative among the four species, ranging from 24953 to $25181 \mathrm{bp}$, but there was some minor variation present in the IR region among the species (Fig. 3). In angiosperms, the downstream sequences of IRb/SSC are mostly conserved, and the $n d h \mathrm{~F}$ gene is adjacent to it (Raubeson et al. 2007), but in $A$. lappa, the $n d h \mathrm{~F}$ gene was located downstream of the IRa/LSC border, which was different from the other species. The border between IRa and LSC regions in the four species (A. lappa, A. tomentosum, C. $\times$ morifolium, and $C$. indicum) was located inside the rps 19 gene, which was different from Lonicera japonica (He et al. 2017), which border between IRa and LSC regions is located inside $r p l 23$. The rps 19 genes of the four species were $279 \mathrm{bp}$ long, and their extension areas to IRa were similar in size $(59-61 \mathrm{bp})$. The IRa region extends into the $y c f 1$ gene, and the $y c f 1$ and $n d h \mathrm{~F}$ genes overlap in A. lappa. In $C$. $\times$ morifolium and $C$. indicum, $y c f 1$ was a cross-border gene with a portion in both the IRa region and the SSC region. Because IRa and IRb had the same gene, they both contained a part of the $y c f 1$ gene, however, the other part of $y c f 1$ was present only in one end of the LSC region, which was close to the IRa/LSC border, but not the other, which was close to the LSC/IRb border. Therefore, the part of $y c f 1$ in the IRb area could not constitute the complete gene and produce vacancies of $884 \mathrm{bp}(C$. indicum $)$ and 886 bp $(C$. $\times$ morifolium $)$ within the IRb. The $t r n \mathrm{H}$ genes were located in the LSC region and the distance from the IRa/LSC border were different, the smallest was 0 bp in C. indicum. The IR region may be highly conserved within the genus, with a high homology within the family that we

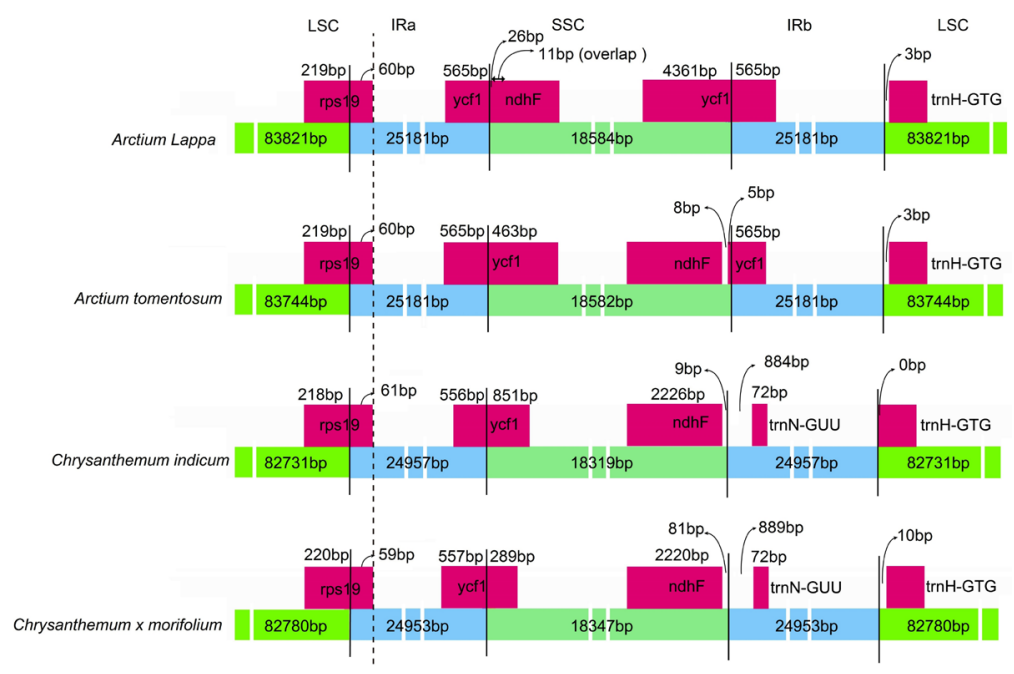

Fig. 3. Schematic representations of large single copies (LSC), small single copies (SSC), and inverted repeat (IRs) border regions in Arctium spp. and Chrysanthemum spp. 


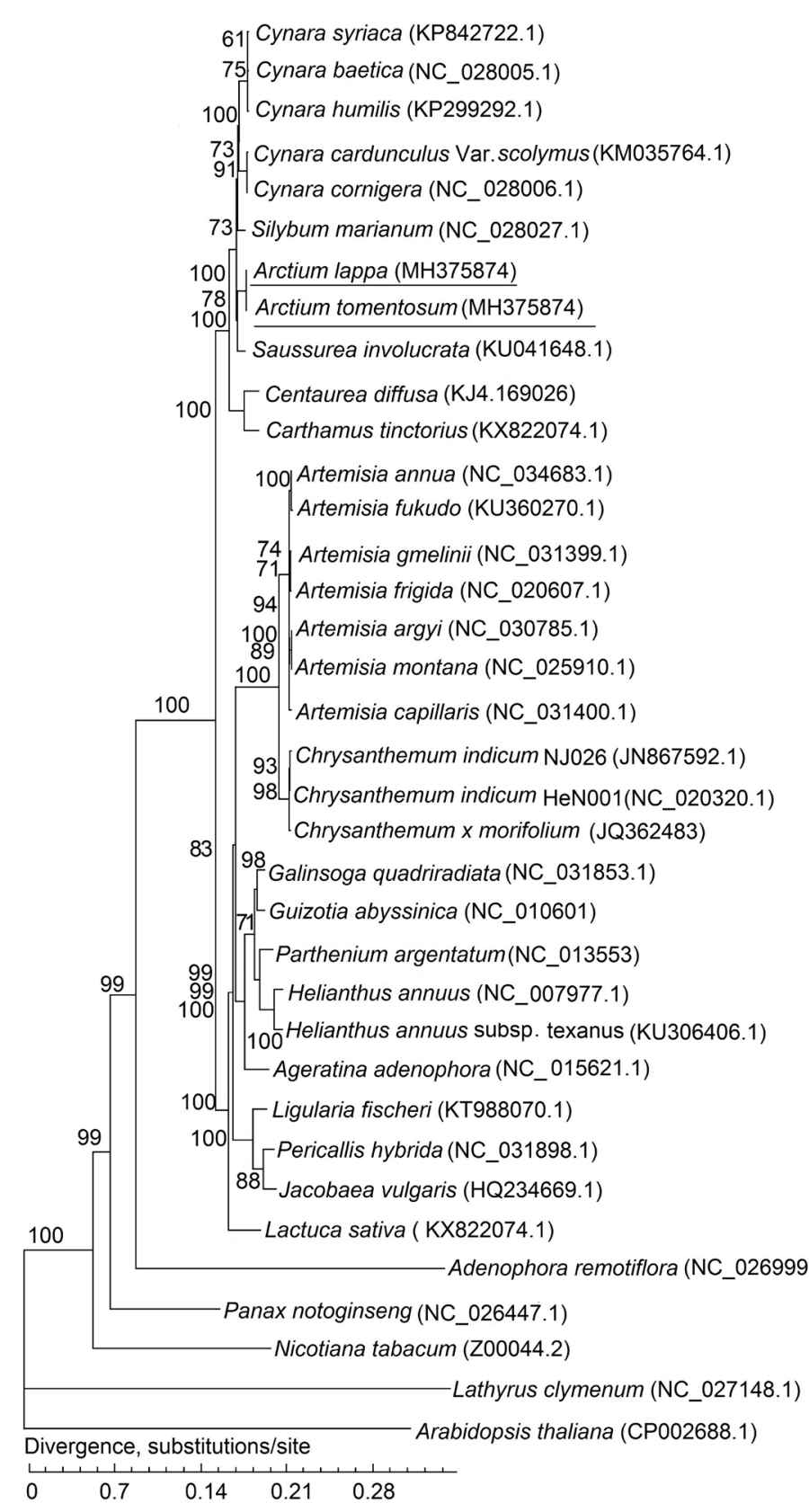

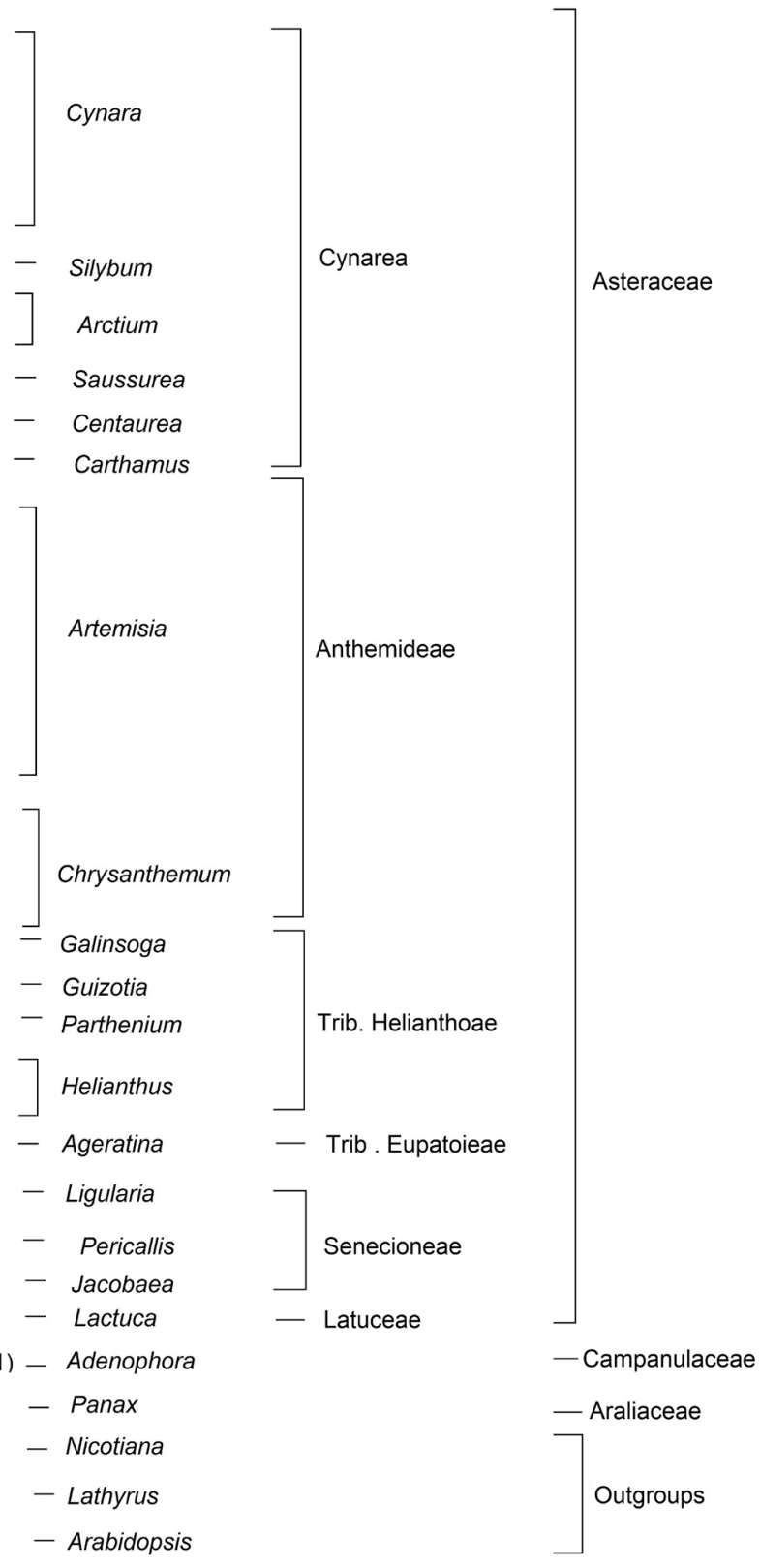

Fig. 4. A molecular phylogenetic tree of 36 species of whole genome single nucleotide polymorphisms (SNPs). The tree was constructed using a maximum likelihood analysis using PhyML 3.0. The model GTR $+\mathrm{I}+\mathrm{G}$ was selected for maximum-likelihood analyses with 100 bootstrap replicates to calculate the bootstrap values.

study, and the IR region was a relatively conserved region due to the ability to correct replication after mutation (Yao et al. 2015).

The overall sequences of the chloroplast genomes of the four Asteraceae species, A. lappa, A. tomentosum, $C$. indicum, and C. $\times$ morifolium, were presented using mVISTA with Silybum marianum (NC_028027.1) as a reference (Fig. 2 Suppl.). The cp genomes in Arctium and Chrysanthemum were similar, but some differences between the two genera were obvious. In Arctium, the divergent regions were between the genes $a t p \mathrm{H}$ and $a t p \mathrm{~F}$ and the genes $p s b \mathrm{Z}$ and $r p s 14$, which were non-coding regions. These different regions could be developed as molecular makers for the identification and phylogenetic analysis.

Because cp genes are conserved within species but vary greatly across species, they are often used for phylogenetic analyses. A phylogenetic tree constructed from genomewide population SNPs (Fig. 4) shows that there was a total of 33 nodes with 13 nodes $100 \%$ supported and 


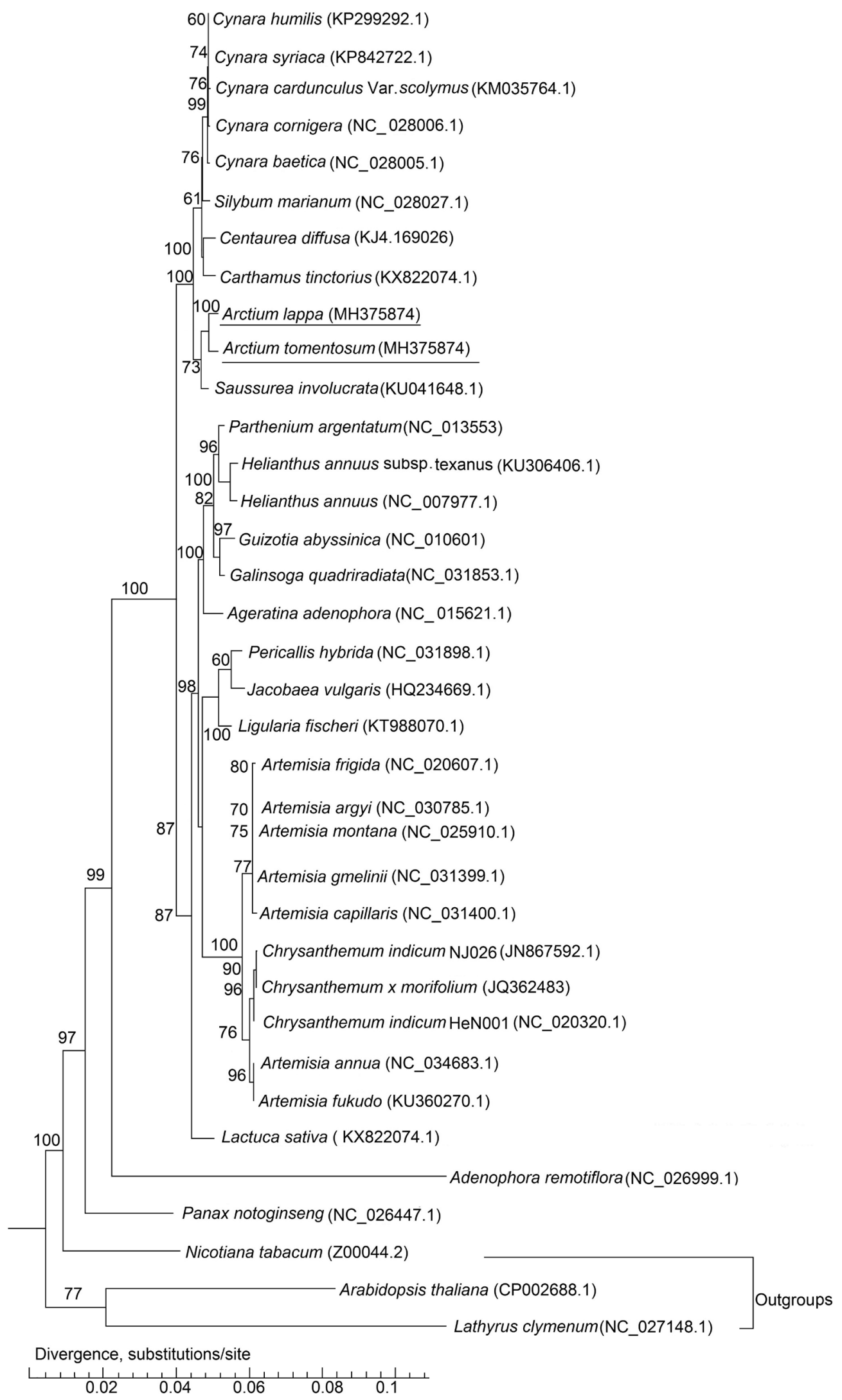

Fig. 5. A molecular phylogenetic tree of 36 species based on 25 shared protein-coding genes in the chloroplast genome. The tree was constructed using a maximum likelihood analysis using PhyML 3.0. The model GTR $+\mathrm{I}+\mathrm{G}$ was selected for ML analyses with 100 bootstrap replicates to calculate the bootstrap values. 
8 nodes $\geq 90 \%$ supported. Four genera of Cynareae, Arctium, Saussurea, Silybum, and Cynara, all clustered into one clade. These four genera are closely related to Certaurea and Carthamus. In addition, Arctium is most closely related to Saussurea. Other Asteraceae species were distributed in Lactuceae, Chrysantheminae, trib. Heliantheae, and tribs. Eupatorieae and Senecioneae. Among them, the trib. Heliantheae and trib. Eupatorieae were close. The other two families, Campanulaceae and Araliaceae, gathered in one clade. All the 31 species in the Asteraceae family clustered in one clade, which supports a monophyly of the Asteraceaeand is similar to previous studies (Wang et al. 2015). Using the SNPs from the cp genome to analyze the phylogenetic relationships among a selection of Asteraceae spp. shows the relative conservatism of Asteraceae cp genes.

Another phylogenetic tree (Fig. 5) was constructed from a total of 25 shared protein-coding sequences for the analysis of 36 species, requiring protein similarity threshold values $>40 \%$. The number of tree nodes was 34 , and there were 18 nodes with $\geq 90 \%$ support and 9 nodes with $100 \%$ support. In this tree, Arctium was a sister to Saussurea and Silybum as well as five Cynara species. Seven Artemisia species composed one clade, which was a sister to Chrysanthemum. This is different from the tree based upon SNPs. The position analysis of A. lappa and $A$. tomentosum by two ways are consistent within Asteraceae and with the result according to Chinese traditional morphological classification.

\section{Conclusions}

We obtained the complete $\mathrm{cp}$ genome sequences from A. lappa and A. tomentosum, and the chloroplasts had similar genome sizes. The cp genomes from the Arctium spp. were compared with two Chrysanthemum spp., and the genome size was also found to be similar. There were some differences in the type and location of genes annotated in $A$. lappa and $A$. tomentosum, which may be suitable for developing markers and conducting phylogenetic analysis. In addition, there were only mononuleotide and dinucleotide SSRs, and those had a similar abundance in the two Chrysanthemum spp. In A. lappa and A. tomentosum, 46 and 36 pairs of large repeat sequences were found. These repeat motifs can be used to develop markers and analyze a phylogenetic tree. A. lappa and A. tomentosum had the same IR lengths and similar with the Chrysanthemum spp. Based on the SNPs from the chloroplast genome and shared coding-proteins, a phylogenetic analysis was conducted to illustrate the position of A. lappa and A. tomentosum in Asteraceae.

\section{References}

Altschul, S.F., Gish, W., Miller, W., Myers, E.W., Lipman, D.J.: Basic local alignment search tool. - J. mol. Biol. 215: 403410, 1990.

Ashburner, M., Ball, C.A., Blake, J.A., Botstein, D., Butler, H.,
Cherry, J.M., Davis, A.P., Dolinski, K., Dwight, S., Eppig, J., Harris, M., Hill, D.P., Issel-Tarver, L., Kasarskis, A., Lewis, S., Matese, J., Richardson, J.E., Ringwald, M., Rubin, G.M., Sherlock, G.: Gene ontology: tool for the unification of biology. - Nat. Genet. 25: 25-29, 2000.

Borgstrom, E., Lundin, S., Lundeberg, J.: Large scale library generation for high throughput sequencing. - PLoS ONE 6: e19119, 2011.

Chan, Y.S., Cheng, L.N., Wu, J.H., Chan, E., Kwan, Y.W., Lee, S.M., Leung, G.P., Yu, P.H., Chan, S.W.: A review of the pharmacological effects of A. lappa (burdock). Inflammopharmacology 19: 245-254, 2011.

Choi, K.S., Park, S.: The complete chloroplast genome sequence of Aster spathulifolius (Asteraceae); genomic features and relationship with Asteraceae. - Gene 572: 214-221, 2015.

Chumley, T.W., Palmer, J.D., Mower, J.P., Fourcade, H.M., Calie, P.J., Boore, J.L., Jansen, R.K.: The complete chloroplast genome sequence of Pelargonium $\times$ hortorum: organization and evolution of the largest and most highly rearranged chloroplast genome of land plants. - Mol. Biol. Evol. 23: 2175-2190, 2006.

Chung, S.M., Deena S.D.W., Jack, E.S.: Genetic relationships within the Cucurbitaceae as assessed by consensus chloroplast simple sequence repeats (ccSSR) marker and sequence analyses. - Can. J. Bot. 81: 814-832, 2003.

Conea, S., Mogoan, C., Vostinaru, O., Toma, C.C., Hepcal, I.C., Cazacu, I., Pop, C., Vlase., L.: Polyphenolic profile, antiinflammatory and antinociceptive activity of an extract from A. lappa L. roots. - Not. Bot. Hort. Agrobot. 45: 59-64, 2017.

Cronn, R., Liston, A., Parks, M., Gernandt, D.S., Shen, R.K.: Multiplex sequencing of plant chloroplast genomes using Solexa sequencing-by- synthesis technology. - Nucl. Acids Res. 36: e122, 2008.

Curci, P.L., De, P.D., Danzi, D., Vendramin, G.G., Sonnante, G.: Complete chloroplast genome of the multifunctional crop globe artichoke and comparison with other Asteraceae. PLoS ONE 10: e0120589, 2015.

Frazer, K.A., Pachter, L., Poliakov, A., Rubin, E.M., Dubchak, A.I.: VISTA: computational tools for comparative genomics. - Nucl. Acids Res. 32: W273-W279, 2004.

He, L., Qian, J., Li, X.W., Sun, Z.Y., Xu, X.L., Chen, S.L.: Complete chloroplast genome of medicinal plant Lonicera japonica: genome rearrangement, intron gain and loss, and implications for phylogenetic studies. - Molecules 22: 249, 2017.

Jansen, R.K., Raubeson, L.A., Boore, J.L., De Pamphilis, C.W., Chumley, T.W., Haberle, R.C., Wyman, S.K., Alverson, A.J., Peery, R., Herman, S.J., Fourcade, H.M., Kuehl, J.V., McNeal, J.R., Leebens M.J., Cui, L.: Methods for obtaining and analyzing whole chloroplast genome sequences. - Methods Enzymol. 395: 348-384, 2005.

Jiao, Y., Jia, H.M., Li, X.W., Chai, M.L., Jia, H.J., Chen, Z., Wang, G.Y., Chai, C.Y., Van de Weg, E., Gao, Z.S.: Development of simple sequence repeat (SSR) markers from a genome survey of Chinese bayberry (Myrica rubra). - BMC Genomics 13: 201, 2012.

Kanehisa, M., Goto, S., Kawashima, S., Okuno, Y., Hattori, M.: The KEGG resource for deciphering the genome. - Nucl. Acids Res. 32: D277-D280, 2004.

Kanehisa, M., Goto, S., Hattori, M., Aoki-Kinoshita, K.F., Itoh, M., Kawashima, S., Katayama, T., Araki, M., Hirakawa, M.: From genomics to chemical genomics: new developments in KEGG. - Nucl. Acids Res. 34: D354-D357, 2006.

Kanehisa, M.: A database for post-genome analysis. - Trends Genet. 13: 375, 1997

Kang, J.S., Lee, B.Y., Kik, M.: The complete chloroplast genome 
sequences of Lychnwas wilfordii and Silene capitata and comparative analyses with other Caryophyllaceae genomes. - PLoS ONE 12: e0172924, 2017.

Khakhlova, O., Bock, R.: Elimination of deleterious mutations in plastid genomes by gene conversion. - Plant J. 46: 85-94, 2006.

Kim, K.J., Lee, H.L.: Complete chloroplast genome sequences from Korean ginseng (Panax schinseng Nees) and comparative analysis of sequence evolution among 17 vascular plants. DNA Res. 11: 247-261, 2004.

Kim, K.J., Choi, K.S., Jansen, R.K.: Two chloroplast DNA inversions originated simultaneously during the early evolution of the sunflower family (Asteraceae). - Mol. Biol. Evol. 22: 1783-1792, 2005.

Kim, W.J., Moon, B.C., Yang, S., Han, K.S., Choi, G., Lee, A.Y.: Rapid authentication of the herbal medicine plant species Aralia continentalis Kitag., and Angelica bwaserrata C.Q. Yuan and R.H. Shan using ITS2 sequences and multiple x-SCAR markers. - Molecules 21: 270, 2016.

Koren, S., Schatz, M.C., Walenz, B.P., Martin, J., Howard, J., Ganapathy, G., Wang, Z., Rasko, D.A., McCombie, W. R., Jarvis, E.D., Phillippy, A.M.: Hybrid error correction and de novo assembly of single-molecule sequencing reads. - Nat. Biotechnol. 30: 693-700, 2012.

Li, X.W., Gao, H.H., Wang, Y.T., Song, J.Y., Robert, H., Wu, H.Z., Hu, Z.G., Yao, H., Luo, H.M., Luo, K., Pan, H.L., Chen, S.L.: Complete chloroplast genome sequence of Magnolia grandiflora and comparative analysis with related species. Sci. China Life Sci. 56: 189-198, 2013.

Liu, L.P., Wan, Q., Guo, Y.P., Yang, J., Rao, G.Y.: Phylogeny of the genus Chrysanthemum L.: evidence from single-copy nuclear gene and chloroplast DNA sequences. - PLoS ONE 7: e48970, 2012.

Lohse, M., Drechsel O., Kahlau, S., Bock, R.: Organellar Genome DRAW - a suite of tools for generating physical maps of plastid and mitochondrial genomes visualizing expression data sets. - Nucl. Acids Res. 41: W575-W581, 2013.

Luo, R., Liu, B., Xie, Y., Li, Z., Huang, W., Yuan, J., He, G., Chen, Y., Pan, Q., Liu, Y., Tang, J., Wu, G., Zhang, H., Shi, Y., Liu, Y., Yu, C., Wang, B., Lu, Y., Han, C., Cheung D.W., Yiu, S., Peng, S., Zhu, X., Liu, G., Liao, X., Li, Y., Yang, H., Wang, J., Lam T.W., Wang, J.: SOAPdenovo2: an empirically improved memory-efficient short-read de novo assembler. Gigascience 1: 18, 2012.

Masalih, R.H., Majesky, L., Schwarzacher, T., Gornall, R., Heslop-Harrison, P.: Complete chloroplast genomes from apomictic Taraxacum (Asteraceae): Identity and variation between three microspecies. - PLoS ONE 12: e0168008, 2017.

Magrane, M., Consortium, U.: UniProt knowledge base: a hub of integrated protein data database. - Databases (Oxford) 2011: bar009, 2011.

McPherson, H., Van der Merwe, M., Delaney, S.K., Edwards, M.A., Henry, H.R., McIntosh, E., Robert, J.H., McIntosh, E., Rymer, P.D., Milner, M.L., Siow, J., Rossetto, M.: Capturing chloroplast variation for molecular ecology studies: a simple next generation sequencing approach applied to a rainforest tree. - BMC Ecol. 13: 8, 2013.

Ohyama, K., Fukuzai, H., Kohchi, T., Shirai, H., Sano, T., Sano, S., Umesono, K., Shiki,Y., Takeuchi, M., Chang, Z., Aota, S.I., Inokuchi, H., Ozeki, H.: Chloroplast gene organization deduced from complete sequence of liverwort Marchantia polymorpha chloroplast DNA. - Nature 322: 572-574, 1986.

Palmer, J.D., Thompson, W.F.: Chloroplast DNA rearrangements are more frequent when a large inverted repeat sequence was lost. - Cell 29: 537-550, 1982.

Raubeson, L.A., Jansen, R.K.: Chloroplast genomes of plants, plant diversity and evolution: genotypic and phenotypic variation in higher plants. - Mol. Phylogenet. Evol. 3: 45-68, 2005.

Raubeson, L.A., Peery, R., Chumley, T.W., Dziubek, C., Fourcade, H.M., Boore, J.L., Jansen, R.K.: Comparative chloroplast genomics: analyses including new sequences from the angiosperms Nuphar advena and Ranunculus macranthus. - BMC Genomics 8: 174, 2007.

Ravi, V., Khurana, J.P., Tyagi, A.K., Khurana, P.: An update on chloroplast genomes. - Plant Syst. Evol. 271: 101-122, 2008.

Tatusov, R.L., Koonin, E.V., Lipman, D. J.: Agenomic perspective on protein families. - Science 278: 631-637, 1997.

Tatusov, R.L., Fedorova, N.D., Jackson, J.D., Jacobs, A.R., Kiryutin, B., Koonin, E.V., Krylov, D.M., Mazumder, R., Mekhedov, S.L., Nikolskaya, A.N., Bachoti,S., Smirnov,S., Sverdlov, A.V., Vasudevan, S., Wolf, Y.I.,Yin, J.J., Natale, D.A., Yin, J.J., Natale, D.A.: The COG database: an updated version includes eukaryotes. - BMC Bioinformatics 4: 41, 2003.

Tseng, M.C., Wong, S.L., Hsiung, D.S., Hwang, J.H., Lee, S.C., Chen, F.H.: Genetic diversity of the chloroplast $\operatorname{trn} \mathrm{L}-\operatorname{trn} \mathrm{F}$ intergenic spacer and nuclear internal transcribed spacer of great burdock (Arctium Lappa L.) in Taiwan. - J. med. Plants Res. 6: 5086-5093, 2012.

Wang, M., Cui, L., Feng, K., Deng, P., Du, X., Wan, F., Song, W., Nie, X.: Comparative analysis of Asteraceae chloroplast genomes: structural organization, RNA editing and evolution. - Plant mol. Biol. Rep. 33: 1526-1538, 2015.

Wicke, S., Schneeweiss, G.M., De Pamphilis, C.W., Muller, K.F., Quandt, D.: The evolution of the plastid chromosome in land plants: gene content, gene order, gene function. - Plant mol. Biol. 76: 273-297, 2017.

Wills, D.M., Hester, M.L., Liu, A., Burke, J. M.: Chloroplast SSR polymorphisms in the Compositae and the mode of organellar inheritance in Helianthus annuus. - Theor. appl. Genet. 110: 941-947, 2005.

Wu, M.L., Li, Q., Xu, J., Li, X.W.: Complete chloroplast genome of the medicinal plant Amomum compactum: gene organization, comparative analysis, and phylogenetic relationships within Zingiberales. - Chin. Med. 13: 10, 2018.

Wu, Z.Q., Ge, S.: The phylogeny of the BEP clade in grasses revisited: evidence from the whole genome sequences of chloroplasts. - Mol. Phylogenet. Evol. 62: 573-578, 2012.

Wyman, S.K., Jansen, R.K., Boore, J.L.: Automatic annotation of organellar genomes with DOGMA. - Bioinformatics 20: 3252-3255, 2004.

Xu, L., Dou, D.Q., Wan, B., Yang, Y.Y., Kang, T.G., Liu, Y.: [Identification of traditional Medicine Fructus Arctii by nuclear ribosomal DNA ITS sequences.] - China J. Chin. Mater. Med. 36: 45, 2011. [In Chin.]

Yang, J.B., Tang, M., Li, H.T., Zhang, Z.R., Li, D.Z.: Complete chloroplast genome of the genus Cymbidium: lights into the species identification, phylogenetic implications and population genetic analyses. - BMC Evol. Biol. 13: 84, 2013.

Yang, J.B., Yang, S.X., Li, H.T., Yang, J., Li, D.Z.: Comparative chloroplast genomes of Camellia species. - PLoS ONE 8: e73053, 2013.

Yao, X., Tang, P., Li, Z., Li, D., Liu, Y., Huang, H.: The first complete chloroplast genome sequences in Actinidiaceae: genome structure and comparative analysis. - PLoS ONE 10: e0129347, 2015. 